\title{
Banco de sementes do solo após aplicação de herbicidas pré-emergentes durante quatro safras de cana-de-açúcar
}

\author{
Reynaldo Tancredo Amim(1), Silvério de Paiva Freitas ${ }^{(1)}$, \\ Ismael Lourenço de Jesus Freitas ${ }^{(1)}$ e Murilo Francischinelli Scarso ${ }^{(2)}$
}

(1)Universidade Estadual do Norte Fluminense Darcy Ribeiro, Avenida Alberto Lamego, no 2.000, Parque Califórnia, CEP 28013-602 Campos dos Goytacazes, RJ, Brasil. E-mail: reynaldo.amim@iff.edu.br, silverio@uenf.br, ismaelljf@yahoo.com.br ${ }^{(2)}$ Bayer Crop Science, Avenida Braz Olaia Acosta, no 727, Jardim Califórnia, CEP 14026-000 Ribeirão Preto, SP, Brasil. E-mail: murilo.scarso@bayer.com

\begin{abstract}
Resumo - O objetivo deste trabalho foi avaliar o efeito dos herbicidas pré-emergentes indaziflam, indaziflam+metribuzim e diuron+hexazinone sobre o banco de sementes do solo de lavoura de cana-de-açúcar, durante quatro safras consecutivas. Utilizou-se o delineamento experimental de blocos ao acaso, com quatro repetições, em parcelas subdivididas. Os tratamentos consistiram de doses dos seguintes herbicidas: indaziflam, 75, 100 e $150 \mathrm{~g}$ i.a. ha ${ }^{-1}$; indaziflam+metribuzim, $75+960$ g i.a. ha ${ }^{-1}$; e diuron+hexazinone, $936+264$ g i.a. ha ${ }^{-1}$; além de área capinada, como testemunha. $\mathrm{O}$ banco de sementes foi analisado nas camadas do solo de $0,00-0,10$ e $0,10-0,20 \mathrm{~m}$. Realizaram-se a contagem e a identificação de plântulas emergidas, e calcularam-se a densidade, a frequência e a abundância absolutas e relativas, bem como o índice de valor de importância. Nos tratamentos 100 e 150 g i.a. ha ${ }^{-1}$ de indaziflam, houve maior redução do banco de sementes total e do índice de valor de importância das espécies de Poaceae e Asteraceae. O banco de sementes total, na camada de 0,10-0,20 m, e o da espécie Portulaca oleracea, nas duas camadas avaliadas, não são influenciados pelos herbicidas. $\mathrm{O}$ uso dos herbicidas pré-emergentes, durante quatro safras consecutivas, reduz o banco de sementes do solo na camada de 0,0-0,10 m, em lavoura de cana-de-açúcar.
\end{abstract}

Termos para indexação: Saccharum, diuron, hexazinone, indaziflam, metribuzim, plantas daninhas.

\section{Soil seed bank after the application of pre-emergent herbicides during four sugarcane harvests}

\begin{abstract}
The objective of this work was to evaluate the effect of the pre-emergent herbicides indaziflam, indaziflam+metribuzin, and diuron+hexazinone on the soil seed bank of a sugarcane crop, during four consecutive harvests. The experimental design was a randomized complete block with four replicates in a split plot. Treatments consisted of doses of the following herbicides: indaziflam, 75, 100, and $150 \mathrm{~g} \mathrm{a.i.} \mathrm{ha}{ }^{-1}$; indaziflam+metribuzin, $75+960 \mathrm{~g}$ a.i. ha $^{-1}$; and diuron+hexazinone, $936+264 \mathrm{~g}$ a.i. ha ${ }^{-1}$; besides a clean area, used as a reference. The soil seed bank was analyzed in the $0.00-0.10$ and $0.10-0.20-\mathrm{m}$ soil layers. Emerged seedlings were counted and identified, and absolute and relative density, frequency and abundance, as well as the importance value index, were calculated. In the treatments 100 and $150 \mathrm{~g}$ a.i. ha ${ }^{-1}$ indaziflam, there was a greater reduction in the total seed bank and in the importance value index of species of Poaceae and Asteraceae. The total seed bank, in the $0.10-0.20-\mathrm{m}$ layer, and that of the species Portulaca oleracea, in both evaluated layers, are not affected by the herbicides. The use of pre-emergent herbicides during four consecutive harvests decreases the soil seed bank in the $0.00-0.10-\mathrm{m}$ layer under a sugarcane crop.
\end{abstract}

Index terms: Saccharum, diuron, hexazinone, indaziflam, metribuzin, weeds.

\section{Introdução}

As plantas daninhas apresentam grande capacidade de produção de sementes viáveis e longevas, com germinação de forma descontínua e adaptações especiais para disseminação a curta e a longa distância. Essas características lhes conferem alta rusticidade
(Pitelli, 1987) e contribuem para a formação e a manutenção do banco de sementes do solo. Segundo Carmona (1992), o banco de sementes tem a função básica de substituir as plantas eliminadas por determinada causa natural ou antropogênica, sendo, portanto, crucial para a sobrevivência das espécies. 
O tamanho e a composição botânica da população de sementes no solo, em um dado momento, são resultantes do balanço entre entradas e perdas de novas sementes no sistema. $\mathrm{O}$ grande potencial produtivo de sementes por algumas espécies daninhas, como exemplificado por Brighenti \& Oliveira (2011) e por Brighenti et al. (2007), é um dos principais fatores responsáveis pelo aumento do banco de sementes do solo. Assim, herbicidas com longa ação residual no solo podem interferir na dinâmica de entrada e saída de sementes no sistema, ao controlar sua germinação, o que influencia em médio e longo prazo o número de espécies e indivíduos presentes nele (Kuva et al., 2008), além de prejudicar a principal forma de entrada de sementes no banco, que é a "chuva de sementes" (Carmona, 1992).

Em um sistema produtivo sustentável, o banco de sementes deve ser observado sob os aspectos ecológico e agronômico. Quanto ao aspecto agronômico, devem-se considerar as práticas adotadas no manejo das culturas, do solo e das plantas daninhas, o que torna imprescindível estudos que avaliem o efeito de herbicidas sobre o banco de sementes do solo.

Recentemente, em janeiro de 2016, o indaziflam, um herbicida de baixa solubilidade em água, foi registrado no Brasil, pelo Ministério da Agricultura, Pecuária e Abastecimento, para o controle de plantas daninhas nas culturas de cana-de-açúcar, café e Citrus. O herbicida apresenta elevado período residual no solo, que pode ser superior a 150 dias, e persiste no solo por mais tempo do que outros herbicidas pré-emergentes (Kaapro \& Hall, 2012); contudo, não há conhecimento de trabalho científico que trate do seu efeito sobre o banco de sementes do solo.

O objetivo deste trabalho foi avaliar o efeito dos herbicidas pré-emergentes indaziflam, indaziflam + metribuzim e diuron+hexazinone sobre o banco de sementes do solo de lavoura de cana-deaçúcar, durante quatro safras consecutivas.

\section{Material e Métodos}

O experimento foi realizado no Município de Paulínia, SP (22044'34"S, 4706'24"W), entre 2008 e 2013, em área cultivada coma variedadedecana-de-açúcar(Saccharum spp.) RB867515. Os tratamentos foram compostos por doses dos seguintes herbicidas: indaziflam, 75, 100 e 150 g i.a. ha ${ }^{-1}$; indaziflam+metribuzim, $75+960$ g i.a. ha ${ }^{-1}$; e diuron+hexazinone, $936+264 \mathrm{~g}$ i.a. ha ${ }^{-1}$; além de área capinada, como testemunha. Utilizou-se o delineamento experimental de blocos ao acaso, com quatro repetições. As parcelas, no total de 48, foram compostas de três linhas da cultura com $5 \mathrm{~m}$ de comprimento, espaçadas entre si em 1,5 metros. A área útil foi composta pelas duas entrelinhas, tendo-se deixado $1 \mathrm{~m}$ em cada extremidade das linhas de plantio como bordadura. Os tratamentos foram aplicados repetidamente na mesma parcela durante quatro safras consecutivas, em 3/12/2008, 4/1/2010, 15/3/2011 e 5/9/2012.

Não foi passado cultivador na área, na ocasião das adubações de cobertura, para evitar contaminação entre as parcelas, e também não foi realizado nenhum tipo de irrigação. O solo da área experimental é um Latossolo Vermelho distroférrico (Embrapa, 1999) com as seguintes características químicas: $17 \mathrm{mg}$ $\mathrm{dm}^{-3}$ de $\mathrm{P}$ resina; $1,3 \mathrm{mmol}_{\mathrm{c}} \mathrm{dm}^{-3}$ de $\mathrm{K} ; 23 \mathrm{mmol}_{\mathrm{c}} \mathrm{dm}^{-3}$ de Ca; $7 \mathrm{mmol}_{\mathrm{c}} \mathrm{dm}^{-3}$ de $\mathrm{Mg} ; 1,0 \mathrm{mmol}_{\mathrm{c}} \mathrm{dm}^{-3}$ de Na; $40 \mathrm{mmol}_{\mathrm{c}} \mathrm{dm}^{-3} \mathrm{de} \mathrm{H}+\mathrm{Al} ; 72,3 \mathrm{mmol}_{\mathrm{c}} \mathrm{dm}^{-3}$ de capacidade de troca catiônica; 32,3 $\mathrm{mmol}_{\mathrm{c}} \mathrm{dm}^{-3}$ de soma de bases; $45 \%$ de saturação por bases; $300 \mathrm{~g} \mathrm{~kg}^{-1}$ de argila; $52 \mathrm{~g} \mathrm{~kg}^{-1}$ de areia; $18 \mathrm{~g} \mathrm{~kg}^{-1}$ de silte; $2,8 \%$ de matéria orgânica; e $\mathrm{pH}$ 6,1. As colheitas foram realizadas de forma manual, sem queima, e a área-testemunha recebeu duas capinas da colheita até o fechamento da cultura.

Para avaliar o efeito dos tratamentos com herbicidas no banco de sementes, foi realizada a coleta do solo nas camadas de $0,0-0,10$ e 0,10-0,20 m, em 22/7/2013, próximo à data de colheita da cana-de-açúcar. $\mathrm{O}$ solo foi coletado com auxílio de trado de caneca com $8,5 \mathrm{~cm}$ de diâmetro (área $=0,00567 \mathrm{~m}^{2}$ ), tendo-se coletado oito subamostras dentro da área útil da parcela, o que totalizou $0,0454 \mathrm{~m}^{2}$ e constituiu amostra composta. Após a coleta, o solo foi levado para a Universidade Estadual do Norte Fluminense Darcy Ribeiro, localizada no Município de Campos dos Goytacazes, RJ, onde foi realizado o experimento em casa de vegetação, para avaliação do banco de sementes do solo, durante seis meses.

As amostras de solo foram homogeneizadas, e metade do seu volume foi utilizado para caracterização do banco de sementes; portanto, para os cálculos, considerou-se apenas a metade da área amostrada nas parcelas, que foi de $0,0227 \mathrm{~m}^{2}$. O solo foi dividido em duas bandejas, cada uma com dimensões de $20 \mathrm{~cm}$ de largura $30 \mathrm{~cm}$ de comprimento $7 \mathrm{~cm}$ de profundidade, 
que juntas formaram uma unidade experimental. As bandejas foram colocadas em casa de vegetação, em 25/7/2013, e mantidas sob cinco irrigações diárias com $1 \mathrm{~mm}$ cada uma, para estimular a germinação. Durante todo o experimento, foram monitoradas a temperatura e a umidade relativa do ar dentro da casa de vegetação, em intervalos de uma hora, com uso do datalogger RHT10 (Extech Instruments, Nashua, NH, EUA), e, em seguida, foi calculada a média de cada uma: $26,4^{\circ} \mathrm{C}$ e $73,5 \%$, respectivamente.

O número de sementes viáveis foi estimado pela soma do número de plântulas emergidas, avaliadas aos 30, 60, 90, 120, 170 e 200 dias. As plântulas foram separadas de acordo com espécie a partir de literaturas especializadas (Lorenzi, 2006; Moreira \& Bragança, 2010), por comparação com plantas daninhas adultas e por consulta a especialistas na área.

Após cada avaliação, o solo era revolvido para estimular a germinação das sementes presentes que não tinham germinado, e, após a quarta avaliação, em 22/11/2013, suspendeu-se a irrigação do solo por 20 dias, para quebrar possíveis dormências. Assim, a quinta avaliação foi feita aos 170 dias e não aos 150 dias.

Para a análise da estrutura da população do banco de sementes, foram calculados os parâmetros densidade, frequência e abundância absolutas e relativas, bem como o índice de valor de importância (IVI), para avaliar a composição florística dos tratamentos (Mueller-Dombois \& Ellenberg, 1974).

$\mathrm{Na}$ análise estatística, quando necessário, os dados foram transformados pela seguinte equação: $X=\sqrt[4]{n+0,5}$, em que $X$ é o número de sementes viáveis após a transformação; $n$ é número de sementes viáveis originalmente identificadas na unidade experimental; e 0,5 é uma constante. Foi utilizado o programa computacional Saeg (Universidade Federal de Viçosa, Viçosa, MG) para a análise de variância, $\mathrm{e}$, nos casos em que o teste $\mathrm{F}$ foi significativo, as médias foram comparadas pelo teste de Tukey, a $5 \%$ de probabilidade. Após as análises, os dados foram novamente transformados e os dados originais apresentados em número de sementes viáveis por hectare.

\section{Resultados e Discussão}

$\mathrm{Na}$ camada de $0,00-0,10 \mathrm{~m}$, em todos os tratamentos, foram identificadas 29 espécies de plantas daninhas, compreendidas em 11 famílias botânicas (Tabela 1). Nos tratamentos indaziflam (75, 100 e $\left.150 \mathrm{~g} \mathrm{ha}^{-1}\right)$, indaziflam+metribuzim e diuron+hexazinone, foram observadas 17, 12, 12, 16 e 13 espécies, respectivamente, e, na área-testemunha, 24 espécies, com destaque para: Digitaria sanguinalis, Eragrostis pilosa, Amaranthus hybridus, Conyza canadensis, Praxelis pauciflora, Gnaphalium coarctatum, Chamaesyce hirta e Phyllanthus tenellus. Os tratamentos indaziflam, indaziflam + metribuzim e diuron+hexazinone proporcionaram redução das populações de plantas das espécies $D$. sanguinalis e E. pilosa (Poaceae), P. pauciflora (Asteraceae), C. hirta (Euphorbiaceae) e P. tenellus (Phyllanthaceae) em comparação à testemunha. No entanto, apenas indaziflam reduziu $C$. canadensis (Asteraceae), que não se diferenciou da testemunha quando foram utilizados os tratamentos indaziflam+metribuzim e diuronthexazinone. A espécie $G$. coarctatum (Asteraceae) apresentou o menor número de indivíduos nos tratamentos 100 e 150 g i.a. ha ${ }^{-1}$ de indaziflam, quando comparado à testemunha e aos herbicidas diuron+hexazinone. Para a espécie A. hybridus, os tratamentos 75 e $150 \mathrm{~g}$ i.a ha ${ }^{-1}$ de indaziflam reduziram em 81,8 e $69,7 \%$, respectivamente, o número de indivíduos em comparação à testemunha, não tendo diferido dos demais tratamentos com o herbicida.

As espécies Pennisetum setosum e Portulaca oleracea também ocorreram em grande número na testemunha; porém, não diferiram nos tratamentos com o herbicida. Ao se considerar o total de indivíduos, o maior número de sementes viáveis foi identificado na área-testemunha, e o menor, com a dose de $150 \mathrm{~g}$ i.a. ha ${ }^{-1}$ de indaziflam, que apresentou resultado similar ao obtido com a dose de 100 g i.a. ha ${ }^{-1}$ do mesmo herbicida (Tabela 1).

O maior período residual do indaziflam (Alonso et al., 2011; Perry et al., 2011; Kaapro \& Hall, 2012), quando comparado ao dos outros herbicidas utilizados, provavelmente proporcionou controle mais eficaz das plantas daninhas até que a cultura da cana-de-açúcar se estabelecesse. Dessa forma, é esperada redução do banco de sementes das plantas daninhas, em razão da inibição da principal forma de entrada de sementes no sistema, que é a "chuva de sementes" (Carmona, 1992). 
Tabela 1. Número de sementes viáveis por metro quadrado das espécies identificadas pelo método de número de plantas emergidas em casa de vegetação, na camada de $0,00-0,10 \mathrm{~m}$ de solo submetido a tratamento com herbicidas pré-emergentes, durante quatro safras de cana-de-açúcar (Saccharum spp.) ${ }^{(1)}$.

\begin{tabular}{|c|c|c|c|c|c|c|c|}
\hline Classe / Família / Espécie & Testemunha & $\begin{array}{c}\text { IND } \\
\left(75 \mathrm{~g}^{2} . \mathrm{h} \mathrm{ha}^{-1}\right) \\
\end{array}$ & $\begin{array}{c}\text { IND } \\
\left(100 \text { g i.a. ha }{ }^{-1}\right) \\
\end{array}$ & $\begin{array}{c}\text { IND } \\
\left(150 \text { g i.a. ha }{ }^{-1}\right) \\
\end{array}$ & IND+MET & DIU+HEX & $\begin{array}{l}\mathrm{CV} \\
(\%) \\
\end{array}$ \\
\hline \multicolumn{8}{|l|}{ Liliopsida } \\
\hline \multicolumn{8}{|l|}{ Poaceae } \\
\hline Cenchrus echinatus & $44,1 \mathrm{a}$ & $0,0 \mathrm{a}$ & $0,0 \mathrm{a}$ & $11,0 \mathrm{a}$ & $0,0 \mathrm{a}$ & $0,0 \mathrm{a}$ & 25,4 \\
\hline Digitaria sanguinalis & $495,6 \mathrm{a}$ & $33,0 \mathrm{~b}$ & $11,0 \mathrm{~b}$ & $22,0 \mathrm{~b}$ & $33,0 \mathrm{~b}$ & $132,2 b$ & 49,6 \\
\hline Eragrostis pilosa & $881,1 \mathrm{a}$ & $77,1 \mathrm{~b}$ & $44,1 \mathrm{~b}$ & $11,0 \mathrm{~b}$ & $77,1 \mathrm{~b}$ & $44,1 \mathrm{~b}$ & 53,9 \\
\hline Pennisetum setosum & $440,5 \mathrm{a}$ & $187,2 \mathrm{a}$ & $44,1 \mathrm{a}$ & $33,0 \mathrm{a}$ & $187,2 \mathrm{a}$ & $143,2 \mathrm{a}$ & 70,7 \\
\hline Eleusine indica & $22,0 \mathrm{a}$ & $0,0 \mathrm{a}$ & $0,0 \mathrm{a}$ & $0,0 \mathrm{a}$ & $22,0 \mathrm{a}$ & $22,0 \mathrm{a}$ & 44,6 \\
\hline Panicum maximum & $11,0 \mathrm{a}$ & $0,0 \mathrm{a}$ & $0,0 \mathrm{a}$ & $11,0 \mathrm{a}$ & $0,0 \mathrm{a}$ & $0,0 \mathrm{a}$ & 17,1 \\
\hline \multicolumn{8}{|l|}{ Cyperaceae } \\
\hline Cyperus rotundus & $0,0 \mathrm{a}$ & $11,0 \mathrm{a}$ & $0,0 \mathrm{a}$ & $11,0 \mathrm{a}$ & $22,0 \mathrm{a}$ & $231,3 \mathrm{a}$ & 53,9 \\
\hline \multicolumn{8}{|l|}{ Magnoliopsida } \\
\hline \multicolumn{8}{|l|}{ Amaranthaceae } \\
\hline Amaranthus hybridus & $363,4 a$ & $66,1 \mathrm{~b}$ & $176,2 \mathrm{ab}$ & $110,1 b$ & $143,2 \mathrm{ab}$ & $187,2 \mathrm{ab}$ & 54,1 \\
\hline \multicolumn{8}{|l|}{ Asteraceae } \\
\hline Conyza canadensis & $231,3 \mathrm{a}$ & $22,0 \mathrm{~b}$ & $22,0 \mathrm{~b}$ & $0,0 \mathrm{~b}$ & $121,1 \mathrm{ab}$ & $99,1 \mathrm{ab}$ & 54,2 \\
\hline Emilia fosbergii & $11,0 \mathrm{a}$ & $11,0 \mathrm{a}$ & $11,0 \mathrm{a}$ & $0,0 \mathrm{a}$ & $22,0 \mathrm{a}$ & $0,0 \mathrm{a}$ & 32,8 \\
\hline Praxelis pauciflora & $286,3 a$ & $0,0 \mathrm{~b}$ & $0,0 \mathrm{~b}$ & $0,0 \mathrm{~b}$ & $0,0 \mathrm{~b}$ & $0,0 \mathrm{~b}$ & 43,3 \\
\hline Gnaphalium coarctatum & $539,6 \mathrm{a}$ & $99,1 \mathrm{bc}$ & $44,1 \mathrm{c}$ & $0,0 \mathrm{c}$ & $110,1 \mathrm{ab}$ & $242,3 \mathrm{ab}$ & 55,0 \\
\hline Bidens pilosa & $44,1 \mathrm{a}$ & $0,0 \mathrm{a}$ & $0,0 \mathrm{a}$ & $0,0 \mathrm{a}$ & $11,0 \mathrm{a}$ & $0,0 \mathrm{a}$ & 28,8 \\
\hline Sonchus oleraceus & $0,0 \mathrm{a}$ & $0,0 \mathrm{a}$ & $0,0 \mathrm{a}$ & $0,0 \mathrm{a}$ & $0,0 \mathrm{a}$ & $11,0 \mathrm{a}$ & 17,1 \\
\hline Vernonia glabrata & $66,1 \mathrm{a}$ & $0,0 \mathrm{a}$ & $0,0 \mathrm{a}$ & $0,0 \mathrm{a}$ & $0,0 \mathrm{a}$ & $0,0 \mathrm{a}$ & 22,1 \\
\hline Porophyllum ruderale & $0,0 \mathrm{a}$ & $11,0 \mathrm{a}$ & $0,0 \mathrm{a}$ & $0,0 \mathrm{a}$ & $0,0 \mathrm{a}$ & $0,0 \mathrm{a}$ & 12,1 \\
\hline Parthenium hysterophorus & $0,0 \mathrm{a}$ & $0,0 \mathrm{a}$ & $0,0 \mathrm{a}$ & $11,0 \mathrm{a}$ & $0,0 \mathrm{a}$ & $0,0 \mathrm{a}$ & 12,1 \\
\hline Baccharis sp. & $11,0 \mathrm{a}$ & $0,0 \mathrm{a}$ & $0,0 \mathrm{a}$ & $0,0 \mathrm{a}$ & $22,0 \mathrm{a}$ & $0,0 \mathrm{a}$ & 22,7 \\
\hline \multicolumn{8}{|l|}{ Euphorbiaceae } \\
\hline Chamaesyce hirta & $33,0 \mathrm{a}$ & $0,0 \mathrm{~b}$ & $0,0 \mathrm{~b}$ & $0,0 \mathrm{~b}$ & $0,0 \mathrm{~b}$ & $0,0 \mathrm{~b}$ & 23,6 \\
\hline \multicolumn{8}{|l|}{ Lamiaceae } \\
\hline Hyptis lophantha & $22,0 \mathrm{a}$ & $11,0 \mathrm{a}$ & $0,0 \mathrm{a}$ & $0,0 \mathrm{a}$ & $0,0 \mathrm{a}$ & $0,0 \mathrm{a}$ & 19,4 \\
\hline \multicolumn{8}{|l|}{ Malvaceae } \\
\hline Sida glaziovii & $11,0 \mathrm{a}$ & $11,0 \mathrm{a}$ & $0,0 \mathrm{a}$ & $0,0 \mathrm{a}$ & $0,0 \mathrm{a}$ & $0,0 \mathrm{a}$ & 20,7 \\
\hline \multicolumn{8}{|l|}{ Oxalidaceae } \\
\hline Oxalis corniculata & $11,0 \mathrm{a}$ & $33,0 \mathrm{a}$ & $33,0 \mathrm{a}$ & $11,0 \mathrm{a}$ & $11,0 \mathrm{a}$ & $0,0 \mathrm{a}$ & 45,2 \\
\hline \multicolumn{8}{|l|}{ Phyllantaceae } \\
\hline Phyllanthus tenelus & $198,2 \mathrm{a}$ & $33,0 \mathrm{~b}$ & $11,0 \mathrm{~b}$ & $11,0 \mathrm{~b}$ & $11,0 \mathrm{~b}$ & $55,1 \mathrm{~b}$ & 49,6 \\
\hline Phyllanthus niruri & $22,0 \mathrm{a}$ & $11,0 \mathrm{a}$ & $0,0 \mathrm{a}$ & $0,0 \mathrm{a}$ & $0,0 \mathrm{a}$ & $0,0 \mathrm{a}$ & 32,5 \\
\hline \multicolumn{8}{|l|}{ Plantaginaceae } \\
\hline Stemodia verticillata & $22,0 \mathrm{a}$ & $0,0 \mathrm{a}$ & $11,0 \mathrm{a}$ & $0,0 \mathrm{a}$ & $0,0 \mathrm{a}$ & $0,0 \mathrm{a}$ & 23,8 \\
\hline \multicolumn{8}{|l|}{ Portulacaceae } \\
\hline Portulaca oleracea & $341,4 a$ & $330,4 a$ & $231,3 a$ & $176,2 \mathrm{a}$ & $352,4 \mathrm{a}$ & $341,4 a$ & 45,9 \\
\hline \multicolumn{8}{|l|}{ Solanaceae } \\
\hline Nicandra physaloides & $55,1 \mathrm{a}$ & $55,1 \mathrm{a}$ & $99,1 \mathrm{a}$ & $11,0 \mathrm{a}$ & $22,0 \mathrm{a}$ & $88,1 \mathrm{a}$ & 58,2 \\
\hline Solanum americanum & $22,0 \mathrm{a}$ & $33,0 \mathrm{a}$ & $0,0 \mathrm{a}$ & $0,0 \mathrm{a}$ & $0,0 \mathrm{a}$ & $33,0 \mathrm{a}$ & 36,3 \\
\hline \multicolumn{8}{|l|}{ Verbenaceae } \\
\hline Lantana camara & $0,0 \mathrm{a} a$ & $0,0 \mathrm{a}$ & $0,0 \mathrm{a}$ & $0,0 \mathrm{a}$ & $33,0 \mathrm{a}$ & $0,0 \mathrm{a}$ & 17,7 \\
\hline Total & $2.312,8 \mathrm{a}$ & $726,9 \mathrm{~b}$ & $638,8 \mathrm{bc}$ & $330,4 \mathrm{c}$ & $859,0 \mathrm{~b}$ & $1.057,3 \mathrm{~b}$ & 26,8 \\
\hline
\end{tabular}


Pode-se inferir que o potencial de infestação das espécies $P$. oleracea e $A$. hybridus foi mantido, uma vez que não houve redução do número de plantas emergidas entre os tratamentos, o que pode ser atribuído às características das sementes dessas espécies, que são pequenas e apresentam superfícies lisas e brilhosas (Groth \& Jamardo, 1982; Coelho \& Giulietti, 2010). De acordo com Vasconcelos et al. (2012), sementes com essas características apresentam dormência, o que explica as suas persistências nos bancos de sementes do solo.

Na camada de 0,10-0,20 m, não houve diferença entre os tratamentos para o total de sementes viáveis presentes no banco de sementes. Entre as espécies, o número de sementes viáveis foi menor para E. pilosa, no tratamento $100 \mathrm{~g}$ i.a. ha ${ }^{-1}$ de indaziflam, que se diferenciou apenas da testemunha, e para A. hybridus, no tratamento indaziflam + metribuzim, que se diferenciou da testemunha e do tratamento 100 g i.a. ha ${ }^{-1}$ de indaziflam (Tabela 2).

Esses resultados podem ser explicados pelos seguintes fatores: baixa mobilidade no solo em que os herbicidas foram utilizados (Inoue et al., 2008; Jhala \& Singh, 2012), o que faz com que atuem nas camadas superficiais, com exceção do hexazinone (Queiroz et al., 2009); menor número de sementes nas camadas mais profundas, em sistemas sem revolvimento do solo (Lacerda et al., 2005); e menor potencial de germinação das sementes situadas nas camadas mais profundas (Vidal et al., 2007; Souza et al., 2009; Monquero et al., 2012), as quais têm pouca influência sobre o banco de sementes.

Houve redução do banco de sementes do solo com o manejo químico, na camada de 0,00-0,10 m, em relação ao tratamento capinado, tanto para as Liliopsidas quanto para as Magnoliopsidas. Para as Magnoliopsidas, houve maior redução da densidade absoluta de sementes viáveis com 150 g i.a. ha-1 de indaziflam, em comparação aos tratamentos diuron+hexazinone e indaziflam + metribuzim, não tendo diferido dos tratamentos 75 e $100 \mathrm{~g}$ i.a. ha ${ }^{-1}$ de indaziflam. Já para as Liliopsidas, os tratamentos 100 e $150 \mathrm{~g}$ i.a. ha ${ }^{-1}$ de indaziflam proporcionaram as menores quantidades de sementes viáveis, que foram inferiores às obtidas no tratamento diuron+hexazinone, mas similares às encontradas nos demais tratamentos químicos. Na camada de $0,10-0,20 \mathrm{~m}$, não houve diferença entre os tratamentos para Liliopsidas e para Magnoliopsidas (Figura 1).

A maior redução do banco de sementes observada para os tratamentos com indaziflam, na camada de 0,00-0,10m,podeseratribuída aomaiorperíodoresidual desse herbicida (Brosnan et al., 2011; Perry et al., 2011). Monquero et al. (2008) verificaram persistência de 60 dias para a mistura diuron+hexazinone $(1.170+330$ g i.a. ha-1). Rocha et al. (2013), por sua vez, relataram meia-vida do diuron isolado (3.000 g i.a. ha ${ }^{-1}$ ) entre 40 e 91 dias, que foi maior no solo com maior teor de matéria orgânica. Quanto ao metribuzim, Fuscaldo et al. (1999) constataram persistência de até 121 dias após aplicação de $960 \mathrm{~g}$ i.a. ha-1.

A redução do banco de sementes verificada especialmente com uso do indaziflam, que apresenta maior período residual, pode ser explicada pelo fato de os herbicidas pré-emergentes proporcionarem controle das plantas daninhas por maior período do que os pós-emergentes (Tompkins, 2010; Kaapro \& Hall, 2012). Posteriormente, o fechamento da cultura e o ciclo longo da cana-de-açúcar proporcionaram controle cultural das plantas daninhas, o que reduziu a produção de novas sementes na área.

$\mathrm{Na}$ testemunha, o número de plantas emergidas variou entre as camadas avaliadas, tendo sido maior na de $0,0-0,10 \mathrm{~m}$, tanto para as Liliopsidas quanto para as Magnoliopsidas, com, respectivamente, 85,15 e $71,43 \%$ do total de indivíduos identificados (Figura 1), provavelmente em decorrência da "chuva de sementes" nessa parcela.

No presente trabalho, o controle frequente das plantas daninhas pelos herbicidas, principalmente o indaziflam nas maiores doses, foi eficaz na redução do banco de sementes do solo, na profundidade de 0,00-0,10 m, o que pode reduzir o potencial de infestação nos próximos ciclos.

O estudo fitossociológico do banco de sementes mostra que, na testemunha, três espécies pertencentes à família Poaceae e duas à Asteraceae apresentaram os maiores IVIs da área, seguidas por $A$. hybridus e $P$. oleracea. Após as aplicações dos herbicidas durante as quatro safras sequenciais de cana-de-açúcar, as Poaceae e as Asteraceae tiveram seus IVIs reduzidos, e as espécies $P$. oleracea e A. hybridus passaram a ter maior importância nas áreas, especialmente nos tratamentos 100 e 150 g i.a. ha ${ }^{-1}$ de indaziflam (Figura 2). 
Tabela 2. Número de sementes viáveis por metro quadrado das espécies identificadas pelo método de número de plantas emergidas em casa de vegetação, na camada de $0,10-0,20 \mathrm{~m}$ de solo submetido a tratamento com herbicidas pré-emergentes, durante quatro safras de cana-de-açúcar (Saccharum spp.).

\begin{tabular}{|c|c|c|c|c|c|c|c|}
\hline Classe / Família / Espécie & Testemunha & $\begin{array}{c}\text { IND } \\
\left(75 \text { g i.a. } \text { ha }^{-1}\right)\end{array}$ & $\begin{array}{c}\text { IND } \\
\left(100 \text { g i.a. ha }{ }^{-1}\right)\end{array}$ & $\begin{array}{c}\text { IND } \\
\left(150 \text { g i.a. ha }{ }^{-1}\right)\end{array}$ & IND+MET & DIU+HEX & $\begin{array}{l}\mathrm{CV} \\
(\%)\end{array}$ \\
\hline \multicolumn{8}{|l|}{ Liliopsida } \\
\hline \multicolumn{8}{|l|}{ Poaceae } \\
\hline Cenchrus echinatus & $11,0 \mathrm{a}$ & $0,0 \mathrm{a}$ & $0,0 \mathrm{a}$ & $0,0 \mathrm{a}$ & $0,0 \mathrm{a}$ & $0,0 \mathrm{a}$ & 25,4 \\
\hline Digitaria sanguinalis & $88,1 \mathrm{a}$ & $11,0 \mathrm{a}$ & $22,0 \mathrm{a}$ & $11,0 \mathrm{a}$ & $11,0 \mathrm{a}$ & $66,1 \mathrm{a}$ & 49,6 \\
\hline Eragrostis pilosa & $132,2 \mathrm{a}$ & $22,0 \mathrm{ab}$ & $11,0 \mathrm{~b}$ & $44,1 \mathrm{ab}$ & $33,0 \mathrm{ab}$ & $22,0 \mathrm{ab}$ & 53,9 \\
\hline Pennisetum setosum & $66,1 \mathrm{a}$ & $11,0 \mathrm{a}$ & $55,1 \mathrm{a}$ & $33,0 \mathrm{a}$ & $66,1 \mathrm{a}$ & $121,1 \mathrm{a}$ & 70,7 \\
\hline Eleusine indica & $33,0 \mathrm{a}$ & $22,0 \mathrm{a}$ & $44,1 \mathrm{a}$ & $22,0 \mathrm{a}$ & $11,0 \mathrm{a}$ & $44,1 \mathrm{a}$ & 44,6 \\
\hline \multicolumn{8}{|l|}{ Commelinaceae } \\
\hline Commelina benghalensis & $0,0 \mathrm{a}$ & $0,0 \mathrm{a}$ & $11,0 \mathrm{a}$ & $0,0 \mathrm{a}$ & $0,0 \mathrm{a}$ & $0,0 \mathrm{a}$ & 12,1 \\
\hline \multicolumn{8}{|l|}{ Cyperaceae } \\
\hline Cyperus rotundus & $0,0 \mathrm{a}$ & $0,0 \mathrm{a}$ & $0,0 \mathrm{a}$ & $308,4 a$ & $0,0 \mathrm{a}$ & $55,1 \mathrm{a}$ & 53,9 \\
\hline \multicolumn{8}{|l|}{ Magnoliopsida } \\
\hline \multicolumn{8}{|l|}{ Amaranthaceae } \\
\hline Amaranthus hybridus & $121,1 \mathrm{~b}$ & $121,1 \mathrm{ab}$ & $341,4 a$ & $297,4 \mathrm{ab}$ & $88,1 \mathrm{~b}$ & $275,3 \mathrm{ab}$ & 54,1 \\
\hline \multicolumn{8}{|l|}{ Asteraceae } \\
\hline Conyza canadensis & $33,0 \mathrm{a}$ & $22,0 \mathrm{a}$ & $11,0 \mathrm{a}$ & $11,0 \mathrm{a}$ & $99,1 \mathrm{a}$ & $0,0 \mathrm{a}$ & 54,2 \\
\hline Emilia fosbergii & $11,0 \mathrm{a}$ & $0,0 \mathrm{a}$ & $11,0 \mathrm{a}$ & $0,0 \mathrm{a}$ & $0,0 \mathrm{a}$ & $11,0 \mathrm{a}$ & 32,8 \\
\hline Praxelis pauciflora & $22,0 \mathrm{a}$ & $0,0 \mathrm{a}$ & $0,0 \mathrm{a}$ & $0,0 \mathrm{a}$ & $0,0 \mathrm{a}$ & $0,0 \mathrm{a}$ & 43,3 \\
\hline Gnaphalium coarctatum & $132,2 \mathrm{a}$ & $121,1 \mathrm{a}$ & $99,1 \mathrm{a}$ & $77,1 \mathrm{a}$ & $77,1 \mathrm{a}$ & $55,1 \mathrm{a}$ & 55,0 \\
\hline Bidens pilosa & $11,0 \mathrm{a}$ & $0,0 \mathrm{a}$ & $0,0 \mathrm{a}$ & $0,0 \mathrm{a}$ & $0,0 \mathrm{a}$ & $11,0 \mathrm{a}$ & 28,8 \\
\hline Sonchus oleraceus & $0,0 \mathrm{a}$ & $0,0 \mathrm{a}$ & $11,0 \mathrm{a}$ & $0,0 \mathrm{a}$ & $0,0 \mathrm{a}$ & $0,0 \mathrm{a}$ & 17,1 \\
\hline Baccharis sp. & $0,0 \mathrm{a}$ & $0,0 \mathrm{a}$ & $0,0 \mathrm{a}$ & $0,0 \mathrm{a}$ & $0,0 \mathrm{a}$ & $11,0 \mathrm{a}$ & 22,7 \\
\hline \multicolumn{8}{|l|}{ Convovulaceae } \\
\hline Ipomoea grandifolia & $0,0 \mathrm{a}$ & $0,0 \mathrm{a}$ & $0,0 \mathrm{a}$ & $0,0 \mathrm{a}$ & $0,0 \mathrm{a}$ & $11,0 \mathrm{a}$ & 12,1 \\
\hline \multicolumn{8}{|l|}{ Euphorbiaceae } \\
\hline Chamaesyce hirta & $0,0 \mathrm{a}$ & $0,0 \mathrm{a}$ & $0,0 \mathrm{a}$ & $0,0 \mathrm{a}$ & $0,0 \mathrm{a}$ & $11,0 \mathrm{a}$ & 23,9 \\
\hline \multicolumn{8}{|l|}{ Lamiaceae } \\
\hline Hyptis lophantha & $0,0 \mathrm{a}$ & $0,0 \mathrm{a}$ & $0,0 \mathrm{a}$ & $0,0 \mathrm{a}$ & $22,0 \mathrm{a}$ & $0,0 \mathrm{a}$ & 19,4 \\
\hline \multicolumn{8}{|l|}{ Malvaceae } \\
\hline Sida glaziovii & $0,0 \mathrm{a}$ & $11,0 \mathrm{a}$ & $0,0 \mathrm{a}$ & $0,0 \mathrm{a}$ & $0,0 \mathrm{a}$ & $0,0 \mathrm{a}$ & 20,7 \\
\hline \multicolumn{8}{|l|}{ Oxalidaceae } \\
\hline Oxalis corniculata & $11,0 \mathrm{a}$ & $33,0 \mathrm{a}$ & $11,0 \mathrm{a}$ & $11,0 \mathrm{a}$ & $22,0 \mathrm{a}$ & $33,0 \mathrm{a}$ & 45,2 \\
\hline \multicolumn{8}{|l|}{ Phyllantaceae } \\
\hline Phyllanthus tenelus & $33,0 \mathrm{a}$ & $22,0 \mathrm{a}$ & $66,1 \mathrm{a}$ & $132,2 \mathrm{a}$ & $44,1 \mathrm{a}$ & $33,0 \mathrm{a}$ & 49,6 \\
\hline Phyllanthus niruri & $0,0 \mathrm{a}$ & $11,0 \mathrm{a}$ & $0,0 \mathrm{a}$ & $44,1 \mathrm{a}$ & $0,0 \mathrm{a}$ & $11,0 \mathrm{a}$ & 32,5 \\
\hline \multicolumn{8}{|l|}{ Plantaginaceae } \\
\hline Stemodia verticillata & $0,0 \mathrm{a}$ & $0,0 \mathrm{a}$ & $0,0 \mathrm{a}$ & $0,0 \mathrm{a}$ & $11,0 \mathrm{a}$ & $0,0 \mathrm{a}$ & 23,8 \\
\hline \multicolumn{8}{|l|}{ Portulacaceae } \\
\hline Portulaca oleracea & $495,6 \mathrm{a}$ & $231,3 a$ & $385,5 \mathrm{a}$ & $374,4 \mathrm{a}$ & $451,5 \mathrm{a}$ & $231,3 a$ & 45,9 \\
\hline \multicolumn{8}{|l|}{ Rubiaceae } \\
\hline Richardia brasiliensis & $0,0 \mathrm{a}$ & $0,0 \mathrm{a}$ & $0,0 \mathrm{a}$ & $0,0 \mathrm{a}$ & $0,0 \mathrm{a}$ & $11,0 \mathrm{a}$ & 12,1 \\
\hline \multicolumn{8}{|l|}{ Solanaceae } \\
\hline Nicandra physaloides & $55,1 \mathrm{a}$ & $88,1 \mathrm{a}$ & $121,1 \mathrm{a}$ & $11,0 \mathrm{a}$ & $297,4 \mathrm{a}$ & $165,2 \mathrm{a}$ & 58,2 \\
\hline Solanum americanum & $0,0 \mathrm{a}$ & $0,0 \mathrm{a}$ & $11,0 \mathrm{a}$ & $0,0 \mathrm{a}$ & $11,0 \mathrm{a}$ & $11,0 \mathrm{a}$ & 17,7 \\
\hline Total & $925,1 \mathrm{a}$ & $660,8 \mathrm{a}$ & $1.068,3 \mathrm{a}$ & $958,1 \mathrm{a}$ & $1.123,3 \mathrm{a}$ & $881,1 \mathrm{a}$ & 26,8 \\
\hline
\end{tabular}

${ }^{(1)}$ Médias seguidas de letras iguais, nas linhas, não diferem pelo teste de Tukey, a 5\% de probabilidade. Dados originais transformados pela equação $X=\sqrt[4]{n+0,5}$. IND, indaziflam; IND+MET, indaziflam+metribuzim; e DIU+HEX, diuron+hexazinone. CV, coeficiente de variação. 


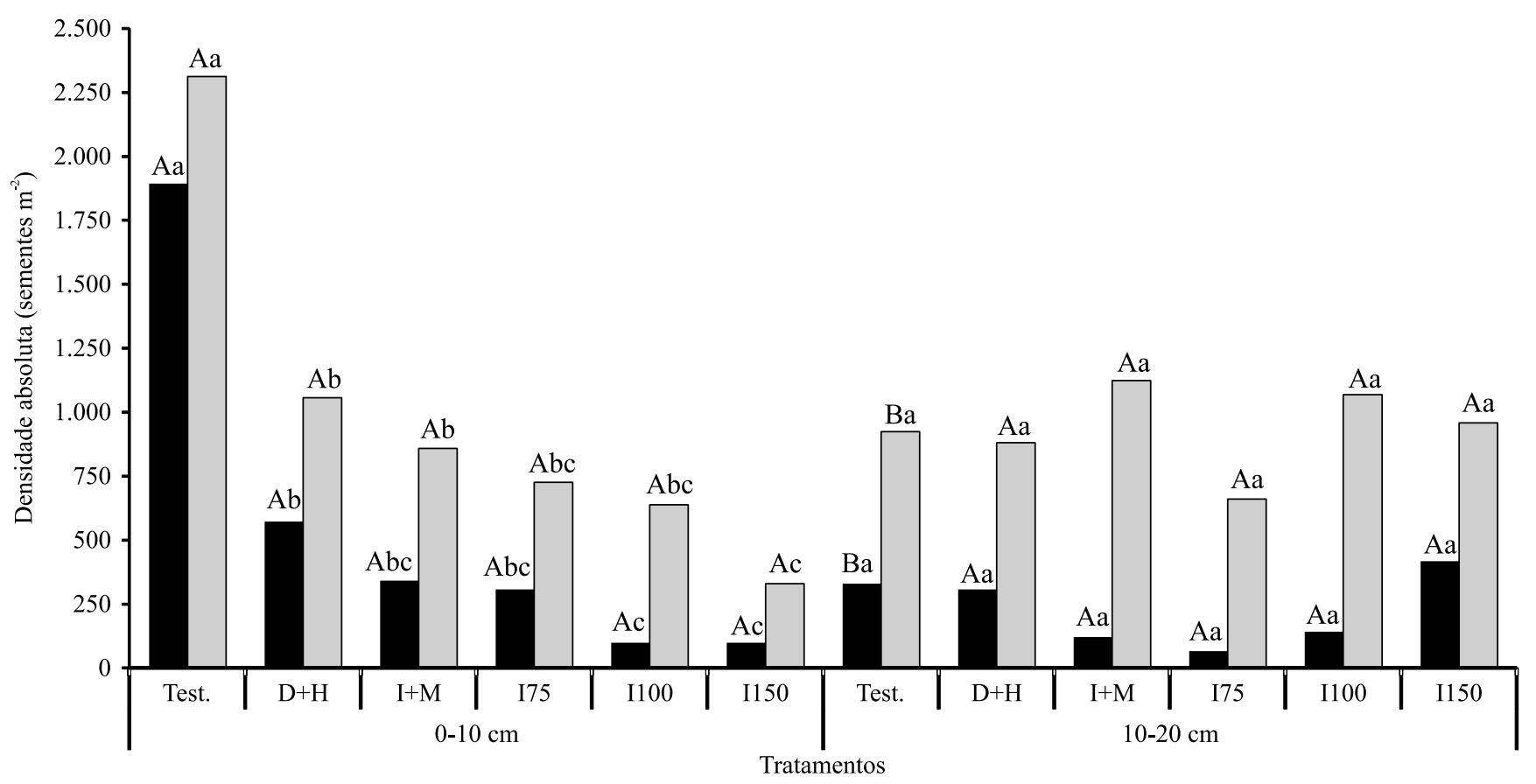

- Liliopsidas $(\mathrm{CV}=55,2 \%) \quad \square$ Magnoliopsidas $(\mathrm{CV}=32,4 \%)$

Figura 1. Densidade absoluta para as classes Liliopsida e Magnoliopsida, no banco de sementes do solo, nas camadas de 0,00-0,10 e 0,10-0,20 m, submetido à aplicação de herbicidas pré-emergentes durante quatro safras de cana-de-açúcar (Saccharum spp.). Testemunha, sem herbicida; D+H, diuron+hexazinone; I+M, indaziflam+metribuzim; I 75, $75 \mathrm{~g}$ i.a. ha ${ }^{-1}$ de indaziflam; I 100, 100 g i.a. ha-1 de indaziflam; e I 150, 150 g i.a. ha ${ }^{-1}$ de indaziflam. Médias seguidas de letras iguais não diferem pelo teste de Tukey, a 5\% de probabilidade. Letras minúsculas comparam os tratamentos em cada profundidade e as maiúsculas, as profundidades em cada tratamento.

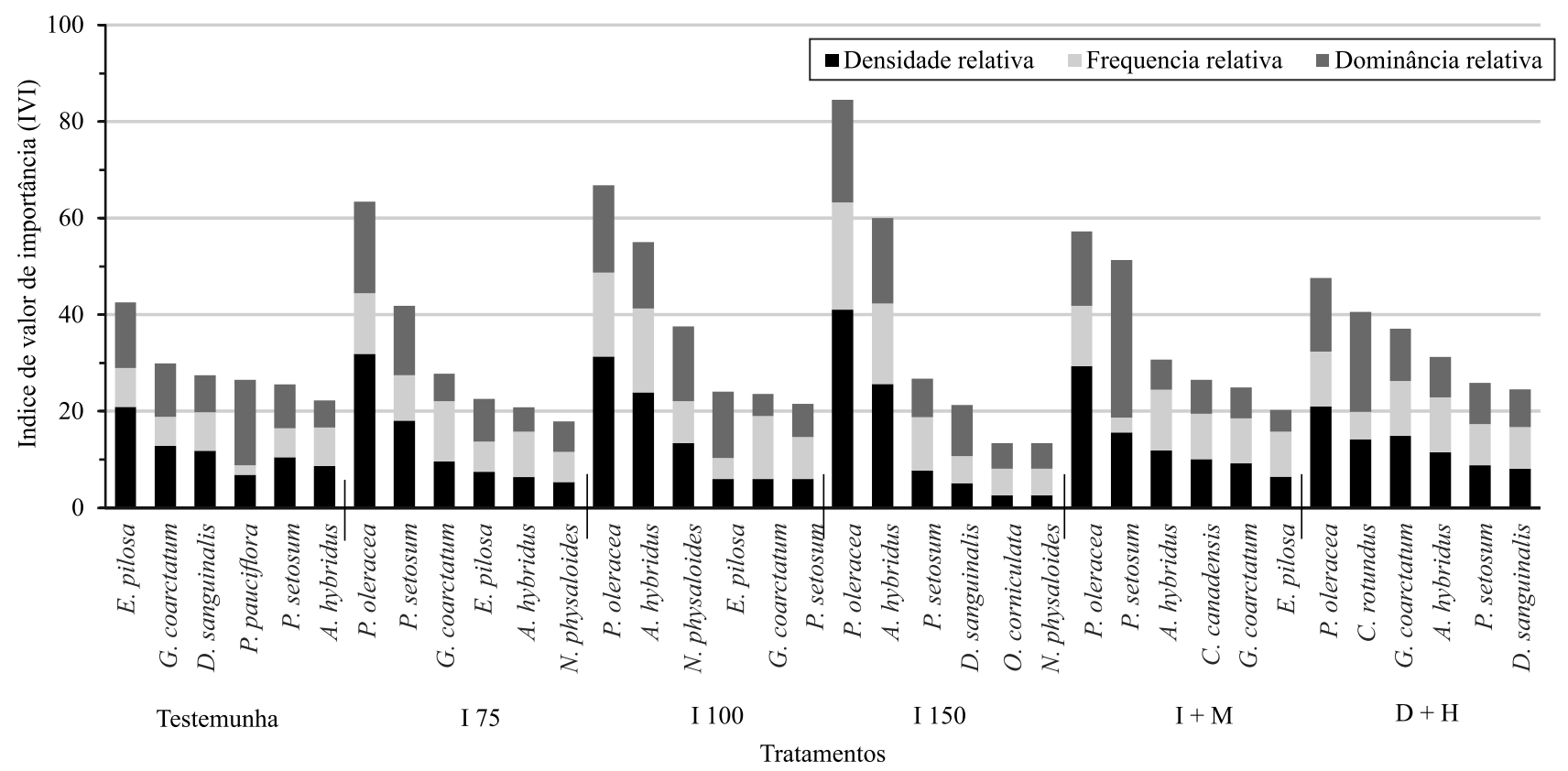

Figura 2. Índice de valor de importância (IVI) das seis principais espécies identificadas no banco de sementes do solo, nas camadas de 0,00-0,10 m, submetido à aplicação de herbicidas pré-emergentes durante quatro safras de cana-de-açúcar (Saccharum spp.). Testemunha, sem herbicida; I 75, 75 g i.a. ha-1 de indaziflam; I 100, 100 g i.a. ha ${ }^{-1}$ de indaziflam; I 150, 150 g i.a. ha ${ }^{-1}$ de indaziflam; I+M, indaziflam+metribuzim; e D+H, diuron+hexazinone. 
Em solo com granulometria semelhante à do presente trabalho - $272 \mathrm{~g} \mathrm{~kg}^{-1}$ de argila, $142 \mathrm{~g} \mathrm{~kg}^{-1}$ de silte e $586 \mathrm{~g} \mathrm{~kg}^{-1}$ de areia -, Amim et al. (2014) mostraram que o indaziflam controlou eficazmente as seguintes espécies da família Poaceae: Digitaria horizontalis, Panicum maximum e Rottboellia cochinchinensis. O controle eficiente de plantas daninhas dessa família, associado ao longo efeito residual do indaziflam (Alonso et al., 2011; Brosnan et al., 2011; Perry et al., 2011), provavelmente influenciou a redução do banco de sementes de espécies da família Poaceae. Correia \& Gomes (2014) ainda observaram que a mistura diuron + hexazinone + sulfometuron proporcionou redução do número de sementes de $R$. cochinchinensis no banco de sementes do solo.

No estudo fitossociológico da camada de $0,10-0,20 \mathrm{~m}$, não houve alteração do banco de sementes pelos herbicidas utilizados no presente trabalho. Entretanto, a espécie $P$. oleracea apresentou os maiores IVIs em quase todos tratamentos, exceto no $150 \mathrm{~g}$ i.a. ha ${ }^{-1}$ de indaziflam, no qual predominou Cyperus rotundus, e no diuron thexazinone, no qual $A$. hybridus teve maior IVI (Figura 3).

O maior IVI observado para $C$. rotundus no tratamento $150 \mathrm{~g}$ i.a. ha ${ }^{-1}$ de indaziflam pode ter resultado do seu alto valor de abundância relativa, o que mostra que houve grande concentração dessa espécie nesse tratamento (Figura 3), provavelmente em razão da presença de reboleiras na área. Cabe ressaltar que as plântulas de C. rotundus identificadas no presente trabalho foram provenientes de tubérculos, os quais foram retirados das bandejas junto com a planta durante as avaliações, para que não houvesse chance desse tubérculo ser contabilizado novamente em futuras avaliações.

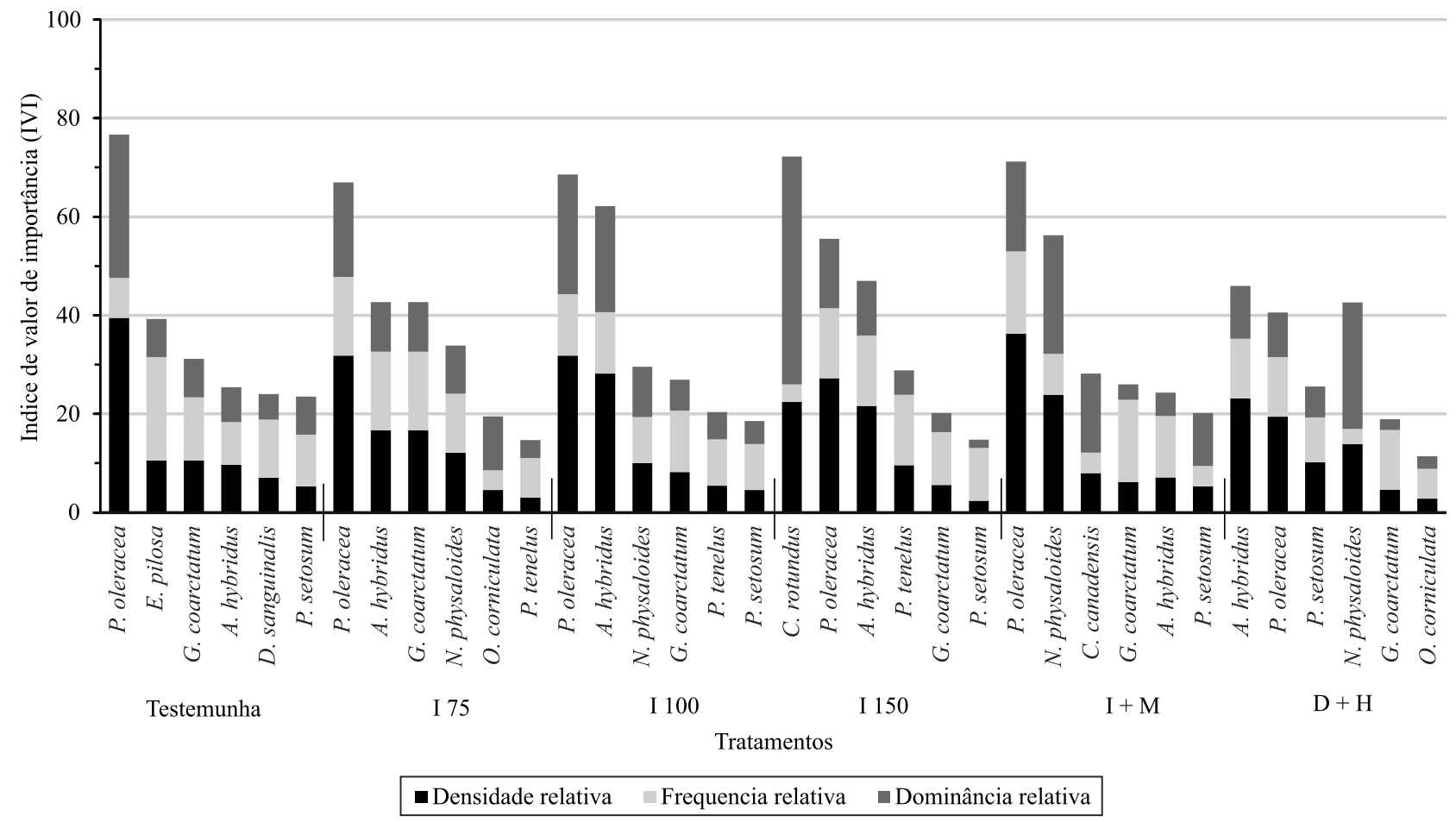

Figura 3. Índice de valor de importância (IVI) das seis principais espécies identificadas no banco de sementes do solo, nas camadas de 0,10-0,20 m, submetido à aplicação de herbicidas pré-emergentes durante quatro safras de cana-de-açúcar (Saccharum spp.). Testemunha, sem herbicida; I 75, 75 g i.a. ha ${ }^{-1}$ de indaziflam; I 100, 100 g i.a. ha-1 de indaziflam; I 150, 150 g i.a. ha ${ }^{-1}$ de indaziflam; $\mathrm{I}+\mathrm{M}$, indaziflam+metribuzim; e D+H, diuron+hexazinone. 


\section{Conclusões}

1. O manejo das plantas daninhas com uso de herbicidas pré-emergentes com maior período residual, durante quatro safras consecutivas de cana-de-açúcar (Saccharum spp.), reduz o banco total de sementes do solo.

2. O banco de sementes total, na camada de 0,10-0,20 m, e o da espécie Portulaca oleracea, nas duas camadas avaliadas, não são influenciados pelos herbicidas pré-emergentes estudados.

3. O indaziflam nas doses de 100 e 150 g i.a. ha ${ }^{-1}$ proporciona maior redução do banco de sementes da espécie Gnaphalium coarctatum.

4. Os herbicidas pré-emergentes estudados são eficazes na redução do banco de sementes das espécies Digitaria sanguinalis, Eragrostis pilosa e Praxelis pauciflora, na camada de $0,0-0,10 \mathrm{~m}$ do solo.

\section{Agradecimentos}

Ao Conselho Nacional de Desenvolvimento Científico e Tecnológico (CNPq), pela concessão de bolsa; e à Bayer, pelo apoio financeiro.

\section{Referências}

ALONSO, D.G.; KOSKINEN, W.C.; OLIVEIRA JR., R.S.; CONSTANTIN, J.; MISLANKAR, S. Sorption-desorption of indaziflam in selected agricultural soils. Journal of Agricultural and Food Chemistry, v.59, p.13096-13101, 2011. DOI: 10.1021/ jf $203014 \mathrm{~g}$.

AMIM, R.T.; FREITAS, S.P.; FREITAS, I.L.J.; GRAVINA, G.A.; PAES, H.M.F. Controle de plantas daninhas pelo indaziflam em solos com diferentes características físico-químicas. Planta Daninha, v.32, p.791-800, 2014. DOI: 10.1590/S010083582014000400014.

BRIGHENTI, A.; VOLL, E.; GAZZIERO, D.L.P. Chloris polydactyla (L.) Sw., a perennial Poaceae weed: emergence, seed production, and its management in Brazil. Weed Biology and Management, v.7, p.84-88, 2007. DOI: 10.1111/j.14456664.2007.00243.x.

BRIGHENTI, A.M.; OLIVEIRA, M.F. Biologia de plantas daninhas. In: OLIVEIRA JR., R.S.; CONSTANTIN, J.; INOUE, M.H. (Ed.). Biologia e manejo de plantas daninhas. Curitiba: Omnipax, 2011. p.1-36.

BROSNAN, J.T.; MCCULlOUGH, P.E.; BREEDEN, G.K. Smooth crabgrass control with indaziflam at various spring timings. Weed Technology, v.25, p.363-366, 2011. DOI: 10.1614/ WT-D-11-00005.1.
CARMONA, R. Problemática e manejo de bancos de sementes de invasoras em solos agrícolas. Planta Daninha, v.10, p.5-16, 1992. DOI: $10.1590 / \mathrm{S} 0100-83581992000100007$.

COELHO, A.A. de O.P.; GIULIETTI, A.M. O gênero Portulaca L. (Portulacaceae) no Brasil. Acta Botanica Brasilica, v.24, p.655-670, 2010. DOI: 10.1590/S0102-33062010000300009.

CORREIA, N.M.; GOMES, L.J.P. Seed bank and control of Rottboellia exaltata using clomazone alone and in combination with other herbicides. Revista Brasilera de Ciência Agrária, v.9, p.538-544, 2014. DOI: 10.5039/agraria.v9i4a4466.

EMBRAPA. Centro Nacional de Pesquisa de Solos (Rio de Janeiro, RJ). Sistema brasileiro de classificação de solos. Brasília: Embrapa-SPI, 1999. 412p.

FUSCALDO, F.; BEDMAR, F.; MONTERRUBBIANESI, G. Persistence of atrazine, metribuzin and simazine herbicides in two soils. Pesquisa Agropecuária Brasileira, v.34, p.2037-2044, 1999. DOI: 10.1590/S0100-204X1999001100009.

GROTH, D.; JAMARDO, A. Caracterização morfológica das unidades de dispersão de seis espécies invasoras da ordem Caryophyllales. Revista Brasileira de Sementes, v.4, p.35-60, 1982. DOI: 10.17801/0101-3122/rbs.v4n3p35-60.

INOUE, M.H.; OLIVEIRA JR., R.S. de; CONSTANTIN, J.; ALONSO, D.G.; SANTANA, D.C. de. Lixiviação e degradação de diuron em dois solos de textura contrastante. Acta Scientiarum. Agronomy, v.30, p.631-638, 2008. DOI: 10.4025/actasciagron. v30i5.5963.

JHALA, A.J.; SINGH, M. Leaching of indaziflam compared with residual herbicides commonly used in Florida citrus. Weed Technology, v.26, p.602-607, 2012. DOI: 10.1614/WT-D-11-00161.1.

KAAPRO, J.; HALL, J. Indaziflam - a new herbicide for preemergent control of weeds in turf, forestry, industrial vegetation and ornamentals. Pakistan Journal of Weed Science Research, v.18, p.267-270, 2012. Special issue.

KUVA, M.A.; PITELLI, R.A.; ALVES, P.L.C.A.; SALGADO, T.P.; PAVANI, M.C.D.M. Banco de sementes de plantas daninhas e sua correlação com a flora estabelecida no agroecossistema cana-crua. Planta Daninha, v.26, p.735-744, 2008. DOI: 10.1590/ S0100-83582008000400004.

LACERDA, A.L.S.; VICTORIA FILHO, R.; MENDONÇA, C.G. Levantamento do banco de sementes em dois sistemas de manejo de solo irrigados por pivô central. Planta Daninha, v.23, p.1-7, 2005. DOI: $10.1590 / \mathrm{S} 0100-83582005000100001$.

LORENZI, H. (Coord). Manual de identificação e controle de plantas daninhas: plantio direto e convencional. 6.ed. Nova Odessa: Instituto Plantarum de Estudos da Flora, 2006. 339p.

MONQUERO, P.A.; HIJANO, N.; ORZARI, I.; SABBAG, R. dos S.; HIRATA, A.C. da S. Profundidade de semeadura, $\mathrm{pH}$, textura e manejo da cobertura do solo na emergência de plântulas de Rottboellia exaltata. Semina: Ciências Agrárias, v.33, p.27992812, 2012. Suplemento 1. DOI: 10.5433/1679-0359.2012v33Sup $11 \mathrm{p} 2799$.

MONQUERO, P.A.; SILVA, A.C.; BINHA, D.P.; AMARAL, L.R.; SILVA, P.V.; INACIO, E.M. Mobilidade e persistência de herbicidas aplicados em pré-emergência em diferentes solos. 
Planta Daninha, v.26, p.411-417, 2008. DOI: 10.1590/S010083582008000200018 .

MOREIRA, H.J. da C.; BRAGANÇA, H.B.N. Manual de identificação de plantas infestantes: cultivos de verão. Campinas: FMC, 2010. 642p.

MUEller-DOMBOiS, D.; ELlENBERG, H. Aims and methods of vegetation ecology. New York: J. Wiley, 1974.

PERRY, D.H.; MCELROY, J.S.; DOROH, M.C.; WALKER, R.H. Indaziflam utilization for controlling problematic turfgrass weeds. Applied Turfgrass Science, v.8, 2011. DOI: 10.1094/ATS2011-0428-01-RS.

PITELLI, R.A. Competição e controle das plantas daninhas em áreas agrícolas. Série Técnica IPEF, v.4, p.1-24, 1987.

QUEIROZ, S.C.N.; FERRACINI, V.L.; GOMES, M.A.F.; e ROSA, M.A. Comportamento do herbicida hexazinone em área de recarga do aquífero Guarani cultivada com cana-de-açúcar. Química Nova, v.32, p.378-381, 2009. DOI: 10.1590/S010040422009000200020 .

ROCHA, P.R.R.; FARIA, A.T.; SILVA, G.S. a; QUEIROZ, M.E.L.R. de; GUIMARÃES, F.C.N.; TIRONI, S.P.; GALON, L.;
SILVA, A.A. da. Meia-vida do diuron em solos com diferentes atributos físicos e químicos. Ciência Rural, v.43, p.1961-1966, 2013. DOI: $10.1590 / \mathrm{S} 0103-84782013001100007$.

SOUZA, M.C.; PITELLI, R.A.; SIMI, L.D.; OLIVEIRA, M.C.J. Emergência de Bidens pilosa em diferentes profundidades de semeadura. Planta Daninha, v.27, p.29-34, 2009. DOI: 10.1590/ S0100-83582009000100005.

TOMPKINS, J. Pesticide fact sheet: indaziflam. 2010. Disponível em: <http://www.epa.gov/opp00001/chem_search/reg_actions/ registration/fs_PC-080818_26-Jul-10.pdf $>$. Acesso em: 30 jan. 2014.

VASCONCELOS, M.C.C. de; SILVA, A.F.A. da; LIMA, R. da S. Interferência de plantas daninhas sobre plantas cultivadas. Agropecuária Científica no Semiárido, v.8, p.1-6, 2012.

VIDAL, R.A.; KALSING, A.; GOULART, I.C.G.R.; LAMEGO, F.P.; CHRISTOFFOLETI, P.J. Impacto da temperatura, irradiância e profundidade das sementes na emergência e germinação de Conyza bonariensis e Conyza canadensis resistentes ao glyphosate. Planta Daninha, v.25, p.309-315, 2007. DOI: 10.1590/ S0100-83582007000200010.

Recebido em 21 de janeiro de 2016 e aprovado em 30 de junho de 2016 